\title{
Low-temperature chemistry using the R-matrix method
}

\begin{abstract}
Jonathan Tennyson, ${ }^{* a}$ Laura K. McKemmish, ${ }^{a}$ and Tom Rivlin ${ }^{a}$
Techniques for producing cold and ultracold molecules are enabling the study of chemical reactions and scattering at the quantum scattering limit, with only a few partial waves contributing to the incident channel, leading to the observation and even full control of state-to-state collisions in this regime. A new R-matrix formalism is presented for tackling problems involving low- and ultra-low energy collisions. This general formalism is particularly appropriate for slow collisions occurring on potential energy surfaces with deep wells. The many resonance states make such systems hard to treat theoretically but offer the best prospects for novel physics: resonances are already being widely used to control diatomic systems and should provide the route to steering ultracold reactions. Our R-matrix-based formalism builds on the progress made in variational calculations of molecular spectra by using these methods to provide wavefunctions for the whole system at short internuclear distances, (a regime known as the inner region). These wavefunctions are used to construct collision energy-dependent $\mathrm{R}$-matrices which can then be propagated to give cross sections at each collision energy. The method is formulated for ultracold collision systems with differing numbers of atoms.
\end{abstract}

\section{Introduction}

To paraphrase the recent review by Stuhl et al., 1 a quiet revolution is occurring at the border between atomic physics and experimental quantum chemistry. There has been a rapid development of techniques for producing cold and even ultracold molecules through techniques such as photoassociation of ultracold alkali atoms, buffer-gas cooling, Stark deceleration, evaporative cooling $^{2}$ and laser cooling ${ }^{3 / 4}$. This progress is now enabling the experimental study of chemical reactions and scattering at the quantum scattering limit with only a few partial waves contributing to the incident channel (e.g. Quemener and Julienne ${ }^{5}$ ). Moreover, the ability to perform these experiments with non-thermal distributions comprising specific states enables the observation and even full control of state-to-state collision rates in this regime. This is perhaps the most elementary study possible of scattering and reaction dynamics. ${ }^{1}$ The trapping ${ }^{6}$ and subsequent study of chemical reactions ${ }^{7}$ involving cold or ultracold chemically important molecules, such as $\mathrm{OH}^{2}$ and $\mathrm{CaH}$, has opened a whole range of possibilities that can be explored in chemical and quantum mechanical control and exploitation. 8 These experimental breakthroughs demand equally transformative theoretical methods for treating ultra cold reactions; these are, for many cases, still lacking.

\footnotetext{
${ }^{a}$ Department of Physics and Astronomy, University College London, London WC1E 6BT, $U K$

*E-mail: j.tennyson@ucl.ac.uk
}

One important feature of ultracold reactions is the pronounced structures present in the cross sections due to temporary formation of long-lived quasi-bound states of the compound system, known as resonances. Resonances are ubiquitous in ultracold collisions ${ }^{9110}$ and offer the best opportunity for quantum control 11 and steering: they are already used to steer the formation of ultracold diatomic molecules: see, for example, Malony et al. ${ }^{[12}$ Furthermore, elastic and inelastic $\frac{13}{13}$ cross sections can dramatically change near resonances, $\frac{14}{14}$ which directly influences the effectiveness of sympathetic cooling and trap losses. These resonances can be manipulated using magnetic and electric fields. ${ }^{[15}$ Studying the structures of resonances in ultracold systems has yielded interesting physics, such as chaos in Dyspronium atoms, 16117 universal scaling laws/ characteristic behaviour 1819 and, when three or more bodies are involved, Efimov resonances. ${ }^{19}$. 27 There are already a number of examples of novel many-body state physics 28 such as Bose-Einstein condensates (BECs), ${ }^{29 \mid 30}$ Efimov trimers, as well as experiments investigating the crossover region between the superfluidity of bosons in BECs and the Cooper pairing of fermions in Bardeen-Cooper-Schrieffer (BCS) theory. $\underline{3132}$

The resonance structure of systems which form over deep wells ${ }^{33}$ in their potentials which support many bound states is likely to be particularly rich and thus offer the greatest potential for transformative science. These deep wells also offer the most opportunity for deviations from previously identified universal characteristics. Here we propose a formalism explicitly designed to study such systems. 
From a theoretical perspective, gas-phase, quantum reactive scattering at room/high temperature is well studied. Timedependent methods have proved to be particularly powerful for these problems 34 . However time-dependent methods struggle at ultra-low collision energies because of the long collision times involved; they are particularly poor at treating resonances. There are time-independent methods available which have been used to treat low-energy collisions. The general physics can often be elucidated using simplified model theories 13

More molecule-specific theories include, in particular, procedures which use hyperspherical coordinates and basis set methods. ${ }^{35 \mid 36}$ These theories have been developed and applied to lowenergy collisions; see Honvault et al 37 , and Pradhan et al. 38 , for example. These methods have been used successfully to treat a number of slow atom-diatom collision problems and are the closest in spirit to what is proposed here. However, the hyperspherical methods generally involve transforming the problem into a series of adiabatic potentials for which solutions are then found. While this approach has proved numerically successful, it is not physically motivated and ultimately involves approximations concerning the couplings between the curves which are hard to overcome.

The idea behind the new proposed R-matrix method for heavy particle scattering is the division of space into two regions: an (energy-independent) inner region where most of the physics takes place, plus an outer region where the interactions are simple. In this inner region, solutions can be obtained by adapting standard bound-state programs. The R-matrix is then constructed on the boundary between these regions. Energy-dependent solutions to the scattering problem can then be obtained rapidly by propagating the R-matrix. First principles, or calculable, R-matrix methods have proved outstandingly successful for studies of light particle collisions 3940 and are being increasingly used in nuclear physics 41 . However, such methods have yet to be systematically applied to heavy particle collisions. R-matrix methods were extensively used to study chemical reaction in the 1980s but, apart from proof-of-principle studies $\frac{42}{2}$, these calculations simply used (outer-region) R-matrix propagation over the entire coordinate range $\mathrm{e}^{43}$. The proposal here is fundamentally different and is much closer in spirit to the methods successfully used by many groups to study electron collisions.

Our R-matrix based formalism builds on the progress made in variational calculations of molecular spectra which are now being used to obtain solutions up to and beyond dissociation for strongly bound systems such as water 4447 and $\mathrm{H}_{3}^{+48,52}$. Both these systems support about a thousand bound vibrational states and many hundreds of thousands of bound rotation-vibration states for which solutions are also being found 53 55. These variational calculations provide wavefunctions for the whole system at short internuclear distances. Indeed, resonances for water 4756 and $\mathrm{H}_{3}^{+[57}$ have already been studied using these approaches and a complex absorbing potential.

There are now a variety of variational nuclear motion methods and related computer programs available for solving these problems. Here we focus on the codes used within our group: specifically the new code Duo, designed for open-shell, coupled-state diatomic problems ${ }^{58}$, DVR3D for three-atom problems ${ }^{59}$ and its four-atom relative WAVR 460 , as well as the general polyatomic code TROVE ${ }^{61 / 62}$. Our group has significant experience with producing spectroscopic accuracy potential energy surfaces that are generally assumed to be essential for quantitative predictions of ultracold collision physics 63 . Hutson $\frac{67}{67}$ presents an interesting counter-argument, demonstrating that if there are significant couplings to inelastic channels, then the sensitivity of the final cross-section to the details of the potential energy surface is reduced as the peaks in the cross-section due to the poles produced by the resonances are suppressed.

In this paper, we present our proposed methodology and illustrate it for the case of atom-atom collisions, utilising the new DuO program $\frac{58}{5}$ to obtain the inner region wavefunctions. This simple system allows a proof-of-principle demonstration of our proposed methodology. Furthermore, the availability of relevant theoretical $^{68}$ and experimental ${ }^{69}$ results will allow thorough benchmarking of our methodology. In particular, we are interested in explaining the surprisingly high measured cross section for the quenching of metastable, excited argon atoms by ultracold argon 69 .

\section{Theoretical background}

Within the Born-Oppenheimer (BO) approximation, the solution of the reactive scattering problem divides into two steps: construction of the global potential energy surfaces using standard quantum chemistry methodologies, and solution of the nuclear motion problem on these surfaces to produce scattering crosssections and other properties of the reaction. We will assume here an appropriate potential energy surface is already available and focus on the second part of this problem. The desired 'solution' for scattering problems is the probability of different processes at a given collision energy. Note that generally, the actual wavefunctions solving the relevant time-independent Schrödinger equation are not necessary; instead, the observable information is embedded in quantities like the phase shifts, scattering $S$ matrix, the $K$ and $T$ matrices and the cross sections.

At large separation between the colliding species, the full scattering problems can be represented in terms of partial waves. The distinguishing characteristic of cold and ultracold scattering problems is that only a small number of these partial wave components are needed to obtain a very good approximation to the full answer. At short separation between the colliding species, a few partial waves are no longer sufficient to describe the physics, particularly when the two species interact strongly, i.e. collide over a potential with a deep well. Instead of trying to use a large number of partial waves, we propose using an approach which treats these two regions separately using methods that are optimal for each region. Specifically, we utilise the powerful variational nuclear motion programs discussed earlier to find collision energy-independent solutions to the inner region problem, $\psi_{k}$, using a single diagonalisation. These energy-independent innerregion wavefunctions are used to construct the so-called R-matrix at the boundary $r=a$ which is given in standard formulations 39 


$$
R_{i, j}(E, a)=\frac{1}{2 a} \sum_{k} \frac{\omega_{k, i} \omega_{k, j}}{E-E_{k}},
$$

where $i$ and $j$ are the asymptotic channels, and $k$ runs over the inner region solutions, and the $\psi_{k}$ functions have energy $E_{k}$ and amplitude on the R-matrix boundary $\underline{\omega}_{k}$. The coordinate $r$ is a radial coordinate which asymptotically goes to dissociation products. Inner region solutions can be obtained explicitly in terms of this coordinate by, for example, working in Jacobi coordinates, or the amplitudes can be obtained by use of a projection operator on the boundary.

Once the R-matrix has been constructed at $r=a$, the energydependent, but computationally simpler, outer region problem is solved to give K-matrices, from which scattering observables, such as cross sections and resonance parameters, can be determined. Due to the computational simplicity of this propagation, this outer-region propagation can be performed on a fine grid of collision energies, essential to elucidate resonance structure. Note that the R-matrix propagation actually becomes simpler at colder temperatures because the number of asymptotic channels decreases significantly. Figure 1 gives a schematic representation of this solution strategy.

\section{Formal theory}

Below we develop the theory needed to solve a simple two atom collision problem on a single potential energy curve. Such a theory might apply to ultracold Ar - Ar collisions. Note that while much of this theory is standard, it is often given in atomic units (assuming electron scattering) 39.40 , such that the reduced mass terms, which are important for heavy particle collisions, are missing.

Treating the inner region as a diatomic system, we can write a molecular ro-vibrational Hamiltonian operator in the following way:

$$
\hat{H}^{J}=\frac{-\hbar^{2}}{2 \mu} \frac{d^{2}}{d r^{2}}+\frac{\hbar^{2} J(J+1)}{2 \mu r^{2}}+\sum_{i \geq i^{\prime}} V_{i i^{\prime}}(r),
$$

where $\mu$ is the reduced mass of the system of two masses $m_{1}$ and $m_{2}$ :

$$
\mu=\frac{m_{1} m_{2}}{m_{1}+m_{2}}
$$

$r$ is the internuclear separation, $J$ is the total angular momentum of the molecule, and $V_{i i^{\prime}}(r)$ is an element of the matrix of potentials associated with the atomic channels, including the offdiagonal channel coupling elements. These couplings can also arise from effects such as spin-orbit interactions which can be represented using a generalisation of rotational operator 70 . At this stage we are interested in both bound and continuum solution to this problem.

Within the R-matrix method a (hyper-)radius $a_{0}$ is chosen where the R-matrix is defined and inner regions solutions are obtained. There is some flexibility over the choice of $\mathrm{a}_{0}$, although our plan is for the inner region to contain regions where the potential well is deep. However we note that the R-matrix method has proved highly successful at finding diffuse, long-range bound states which extend outside the inner region 71 and such states are expected near the dissociation limit of polyatomic systems 51 .
Solving the Schrödinger equation with the Hamiltonian defined in eq. (2) within a finite region requires the introduction of a surface term, $\mathscr{L}$, known as a Bloch term ${ }^{72}$, to retain Hermiticity. The expression for this term is:

$$
\mathscr{L}=\frac{\hbar^{2}}{2 \mu} \delta\left(r-a_{0}\right) \frac{d}{d r},
$$

where $\delta\left(r-a_{0}\right)$ is the standard Dirac delta function. To solve the molecular problem with the surface term, we introduce a set of functions $\left\{\chi_{n}^{J}(r)\right\}$. These functions are obtained as eigensolutions to the matrix problem

$$
\left(\chi_{n}^{J}\left|\left(\hat{H}^{J}+\mathscr{L}\right)\right| \chi_{n^{\prime}}^{J}\right)=E_{n}^{J} \delta_{n n^{\prime}}
$$

where, as is conventional 39 , rounded Dirac brackets have been used to show that integration in the radial coordinate, $r$, only runs over the finite volume of the inner region, from 0 to $a_{0}$. The eigenvalues, $E_{n}^{J}$, of this equation are usually referred to R-matrix poles and their associated eigenfunctions are defined using

$$
\chi_{n}^{J}(r)=\sum_{i} \sum_{j} c_{i j n}^{J} \phi_{i j}^{J}(r)
$$

where $\left\{\phi_{i j}^{J}(r)\right\}$ is some basis, and the coefficients $c_{i j n}^{J}$ are determined by the requirement that eq. (5) is diagonal. Since the $J$ is a conserved quantum number, we may label all solutions with it. Final results require the summation over $J$, but at low energies such sums should converge rapidly.

The indices $i$ and $j$ in eq.6 run over the channels, and the basis functions within each channel. To isolate the contribution from a single channel, one can sum over only the basis functions within that channel, $j$, by defining

$$
w_{i n}(r)=\sum_{j} c_{i j n} \phi_{i j}(r)
$$

From this, elements of the R-matrix, $\mathbf{R}^{J}$, can be defined on the boundary using the heavy particle generalisation of eq. (1)

$$
R_{i i^{\prime}}^{J}\left(E, a_{0}\right)=\frac{\hbar^{2}}{2 \mu a_{0}} \sum_{n} \frac{w_{i n}^{J}\left(a_{0}\right) w_{i^{\prime} n}^{J}\left(a_{0}\right)}{E_{n}^{J}-E},
$$

where $w_{i n}^{J}\left(a_{0}\right)$ is called the surface amplitude (since it is evaluated at the boundary), $E$ is the scattering energy of interest, and the sum is over all $n$, i.e. over all eigensolutions of eq. (5). We note that it is also possible to reformulate the problem to use a reduced set of inner region solutions. ${ }^{73}$ Note that a single set of inner region solutions are used to construct the R-matrix at $r=a_{0}$ for all scattering energies, meaning that the inner region problem only needs to be solved once, independent of how many energies the final solutions are needed for. This is particularly useful for obtaining high-resolution plots of resonances as a function of scattering energy.

From the scattering energy, $E$, the scattering wave number, $k$, can be defined as

$$
k=\frac{\sqrt{2 \mu E}}{\hbar} .
$$

A similar definition exists, and can be obtained from the eigenenergies $E_{n}^{J}$, for the wave numbers $k_{n}^{J}$. These can be written into a 


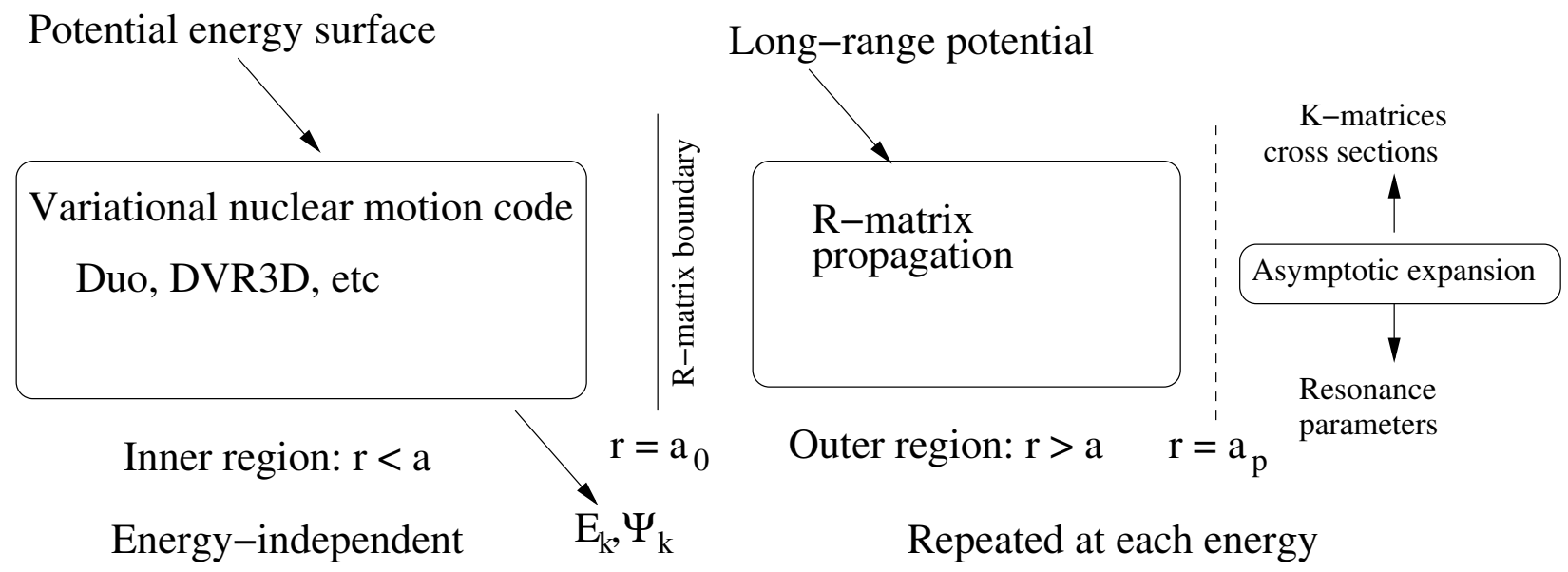

Fig. 1 Schematic division of space illustrating the use of the R-matrix method.

\section{diagonal matrix $\mathbf{k}^{J}$.}

Defining the outer region wavefunctions for a given target channel at some point, $r=a$, as $F_{i}^{J}(a)$, the R-matrix represents the relationship between these functions and their derivatives:

$$
F_{i}^{J}(a)=\left.a \sum_{i^{\prime}} R_{i i^{\prime}}^{J}(E, a) \frac{d F_{i^{\prime}}^{J}(r)}{d r}\right|_{r=a},
$$

where the sum runs over all channels.

Propagating the R-matrix to large $r$ allows the scattering problem to be solved without the explicit need to evaluate the wavefunction which, particularly in the presence of closed channels, can be a difficult task numerically and computationally.

There are a number of means of propagating the R-matrix, including those due to Baluja, Burke and Morgan ${ }^{74 / 75}$, due to Light and Walker 76 , and the software FARM (the flexible asymptotic R-matrix package) $77 / 78$. As discussed below, we favour the use of the Light-Walker propagator. Furthermore, there are several ways of obtaining the asymptotic wavefunctions, $F_{i}^{J}(r)$ 79]80. In this work the asymptotic expansion of Burke and Schey 79 is used. Generally speaking, asymptotic expansions follow the form

$$
F_{i}^{J}(r)=\sum_{i^{\prime}}\left(s_{i i^{\prime}}^{J}\left(k_{i^{\prime}}^{J} r\right)+\sum_{i^{\prime \prime}} c_{i i^{\prime \prime}}^{J}\left(k_{i^{\prime}}^{J} r\right) K_{i^{\prime} i^{\prime \prime}}^{J}(E)\right),
$$

where both sums are over all channels, $K_{i^{\prime} i^{\prime \prime}}^{J}$ is an element of the Kmatrix, $\mathbf{K}^{J}(E)$, and $s_{i i^{\prime}}^{J}$ and $c_{i i^{\prime \prime}}^{J}$ are elements of the matrices $\mathbf{s}^{J}\left(\mathbf{K}^{J} r\right)$ and $\mathbf{c}^{J}\left(\mathbf{k}^{J} r\right)$ respectively. These matrices are matrices of 'sinelike' and 'cosine-like' functions respectively, which are different for different channels. The Burke-Schey asymptotic expansion specifies the form of these functions, and is discussed in detail in the next section.

The propagated R-matrix is then combined with the asymptotic expansion to construct the K-matrix, which has the following form:

$$
\mathbf{K}^{J}(E)=-\frac{\mathbf{s}^{J}\left(\mathbf{k}^{J} r\right)-r \mathbf{R}^{J}(E, r) \dot{\mathbf{s}}^{J}\left(\mathbf{k}^{J} r\right)}{\mathbf{c}^{J}\left(\mathbf{k}^{J} r\right)-r \mathbf{R}^{J}(E, r) \dot{\mathbf{c}}^{J}\left(\mathbf{k}^{J} r\right)},
$$

where $\dot{\mathbf{s}}^{J}\left(\mathbf{k}^{J} r\right)$ and $\dot{\mathbf{c}}^{J}\left(\mathbf{k}^{J} r\right)$ are the derivatives with respect to $r$ of $\mathbf{s}^{J}\left(\mathbf{k}^{J} r\right)$ and $\mathbf{c}^{J}\left(\mathbf{k}^{J} r\right)$ respectively, and $r$ is evaluated at some large value, denoted $a_{p}$.

From the K-matrix, the S- and T-matrices are defined in the following ways:

$$
\begin{aligned}
\mathbf{S}^{J} & =\frac{\mathbf{1}+i \mathbf{K}^{J}}{\mathbf{1}-i \mathbf{K}^{J}} \\
\mathbf{T}^{J} & =\mathbf{S}^{J}-\mathbf{1} .
\end{aligned}
$$

Note that while the definition of the S-matrix is general, the precise definition of the T-matrix depends on the convention adopted.

The eigenphase for each channel, $\delta_{i}^{J}(E)$, is given by the inverse tangent of $\mathbf{K}^{J}(E)$ 's eigenvalues:

$$
\delta_{i}^{J}(E)=\arctan \left(K_{i}^{J}(E)\right),
$$

where $K_{i}^{J}(E)$ is the $i^{\text {th }}$ eigenvalue of the K-matrix, associated with channel $i$. This, in turn, gives the eigenphase sum for a given symmetry $(J)$ :

$$
\delta^{J}(E)=\sum_{i} \delta_{i}^{J}(E)
$$

The total cross section at a given energy, $\sigma_{\text {tot }}(E)$, can be obtained in a number of ways, including from the eigenphase sums:

$$
\sigma_{\mathrm{tot}}(E)=\frac{4 \pi}{k^{2}} \sum_{J=0}^{J_{\max }}(2 J+1) \sin ^{2}\left(\delta^{J}(E)\right),
$$

where $J_{\max }$ is the maximum number of angular momentum values (partial waves) considered. For the ultracold temperatures being considered here, this can be a very small number, possibly a single channel. For multi-channel collisions, the cross section for a transition from channel $i$ to channel $i^{\prime}$ is

$$
\sigma_{i^{\prime} i}(E)=\frac{\pi}{k_{i}^{2}} \sum_{J=0}^{J_{\max }}(2 J+1)\left|T_{i^{\prime} i}^{J}\right|^{2} .
$$

\section{Computational Implementation}

The computational procedure for solving the above equations is essentially made of three steps: (a) the inner region, (b) the boundary and (c) the outer region and asymptopia. The final part, step (c), can be written in a fairly general fashion, which should 
cater for a variety of different systems. Therefore our aim in writing the code which constructs the R-matrix on the boundary, step (b), is to make it rather general to allow for the incorporation of a variety of inner region nuclear motion codes. So far, in practice, we have only used the diatomic code Duo 58 . Duo is designed to compute spectra for open shell diatomic molecules and allows for explicit inclusion of coupled potential energy curves through the inclusion of spin-orbit and other coupling terms. Duo is designed to read in potential energy and coupling curves in a variety of formats, including simply as a grid of points. Here we have used the $\mathrm{Ar}$ - Ar potential of Patkowski et al ${ }^{81}$, defined on a grid. DuO constructs a basis using a Hund's case (a) representation, which is then used to obtain a full variational solution of the problem. Further details and discussion can be found elsewhere $70[82$.

The recently published version of DuO $\frac{58}{58}$ is designed only to treat bound rovibronic states. The first task is therefore to extend this to give wavefunctions for the discretised continuum in the inner region. This is done by constructing a set of functions $\left\{\phi_{i j}^{J}\right\}$. These functions are intended to be the set of square integrable, linearly-independent basis functions which are complete over the $\left[r_{\min }, a_{0}\right]$ range up to some appropriate maximum energy which enter into eq (6). As these functions are used to provide the amplitude of the inner region function $\left\{\chi_{n}^{J}\right\}$ on the boundary at $r=a_{0}$, one has to be careful how these functions behave at this point.

In practice, the basis functions are generated in a two step procedure. An initial basis set, $\left\{\psi_{i j}^{J}\right\}$, is generated by solving the molecular problem associated with the ro-vibrational Hamiltonian, $\hat{H}^{J}$, of eq. (2). In solving this problem, an artificial wall is placed in the potential at some distance $r_{\text {wall }}\left(r_{\text {wall }}>a_{0}\right)$. Tests have shown that use of a wall provides a good representation of resonance states contained inside it, see Fig. 2. Provided the wall is placed far enough out, the $\left\{\psi_{i j}^{J}\right\}$ are effectively computational approximations of the eigenfunctions of $\hat{H}^{J}$, with each basis function index $j$ belonging to a channel $i$. Generally speaking, placing the wall such that $a_{0}$ was approximately $\approx 95 \%$ of the way to $r_{\text {wall }}$ was found to be appropriate $\underline{42}$.

Although the $\left\{\psi_{i j}^{J}\right\}$ basis is constructed by integrating over the full inner region $\left[r_{\mathrm{min}}, r_{\text {wall }}\right]$, the rest of the R-matrix construction method involves integrating over the smaller $\left[r_{\min }, a_{0}\right]$ region. In this region, the $\left\{\psi_{i j}^{J}\right\}$ are not eigenfunctions of the Hamiltonian, so $\mathbf{H}^{J}$, the matrix of the Hamiltonian $\hat{H}^{J}$ in this basis, will not be diagonal when defined over this range. The non-diagonal Hamiltonian matrix is constructed by using a forward finite difference numerical differentiation method of order four to evaluate the kinetic term, and an implementation of Simpson's rule for the numerical integration up to $a_{0}$.

The wall cannot be placed at $a_{0}$ because, by construction, the basis functions have zero amplitude at the wall. At this point there are two possible approaches, both of which have been tested by us.

The earlier proof-of-principle study of an R-matrix approach to reaction dynamics by Bocchetta and Gerratt ${ }^{42}$ simply diagonalised a generalised version of the eigenvalue eq. (5) by including the overlap matrix on the left-hand side. Alternatively, the $\left\{\psi_{i j}^{J}\right\}$ functions can be re-orthonormalised over the $\left[r_{\min }, a_{0}\right]$ range, for which we found symmetric or Löwdin orthonormalisation 83 to be the most suitable. Eventually we decided to utilise a generalised eigenvalue scheme, constructing the $\left\{\chi_{n}^{J}\right\}$ functions directly out of the $\left\{\psi_{i j}^{J}\right\}$. In both methods we constructed a matrix for the Bloch operator in the original basis, $\mathbf{L}$, using the aforementioned forwards finite difference method of order four to compute the derivative at $a_{0}$.

The generalised eigenvalue problem we arrived at was

$$
\left(\mathbf{H}^{J}+\mathbf{L}\right) \chi_{n}^{J}=E_{n}^{J} \mathbf{S}^{J} \chi_{n}^{J},
$$

where $\mathbf{S}^{J}$ is the overlap, or Gramian matrix, whose elements are made of all the possible inner products between the different $\psi_{i j}^{J}$ basis functions. Equation 19 is then solved using the LAPACK 84 routine dsygv to obtain the $\left\{\chi_{n}^{J}\right\}$ and $E_{n}^{J}$ required to construct the R-matrix.

For each value of $J$, our new R-matrix code reads from a version of DuO, adapted to implement the potential wall, all of the eigenenergies and eigenfunctions of the molecular system (the number of which is user-specified in DUO), the minimums $V_{i i \text { min }}$ of the potentials associated with each channel $V_{i i}(r)$, the wall position $r_{\text {wall }}$, the masses of the atoms $m_{1}$ and $m_{2}$, the range over which the eigenfunctions are defined and orthonormalised, $r_{\min }$ and $r_{\max }$, the step size of the integration, $\Delta r$, and the zero point energy (zpe).

This information is used to constuct the R-matrix on the boundary, as outlined above, and this is then propagated outwards using the Light-Walker propagation method to a point $a_{p}$. The LightWalker propagator takes the form of an iteration equation for the R-matrix between the values $a_{0}$ and $a_{p}$, by dividing the region into sub-regions with boundaries $a_{s}$. The propagator is constructed in the following way 39 : we diagonalise the matrix

$$
\mathscr{V}^{J}(r)=\mathbf{V}^{J}(r)-\left(\mathbf{E}^{J}\right)+E \mathbf{I},
$$

where $\mathbf{I}$ is the identity matrix, $E$ is the scattering energy, $\mathbf{E}^{J}$ is the diagonal matrix of eigenenergies (not to be confused with the scattering energy), and $\mathbf{V}^{J}(r)$ is the (in general) non-diagonal matrix of potentials for each channel, including channel coupling elements (defined properly below in eq. (29) - note the $J$-dependence). We call the version of this matrix which has been evaluated at $a_{s}$ and diagonalised $\left(\mathbf{v}_{s}^{J}\right)^{2}$, and the matrix which diagonalises it we call $\mathbf{O}_{s}^{J}$ :

$$
\left(\mathbf{O}_{s}^{J}\right)^{T} \mathscr{V}^{J} \mathbf{O}_{s}^{J}=\left(\mathbf{v}_{s}^{J}\right)^{2}
$$

This allows us to define the real, diagonal matrix $\lambda_{s}^{J}$ in the following way:

$$
\left(\lambda_{s}^{J}\right)^{2}=\frac{2 \mu}{\hbar^{2}}\left(E \mathbf{I}-\left(\mathbf{v}_{s}^{J}\right)^{2}\right)
$$

Next we define elements of the diagonal matrix $\mathscr{G}_{S}^{J}$, made up 


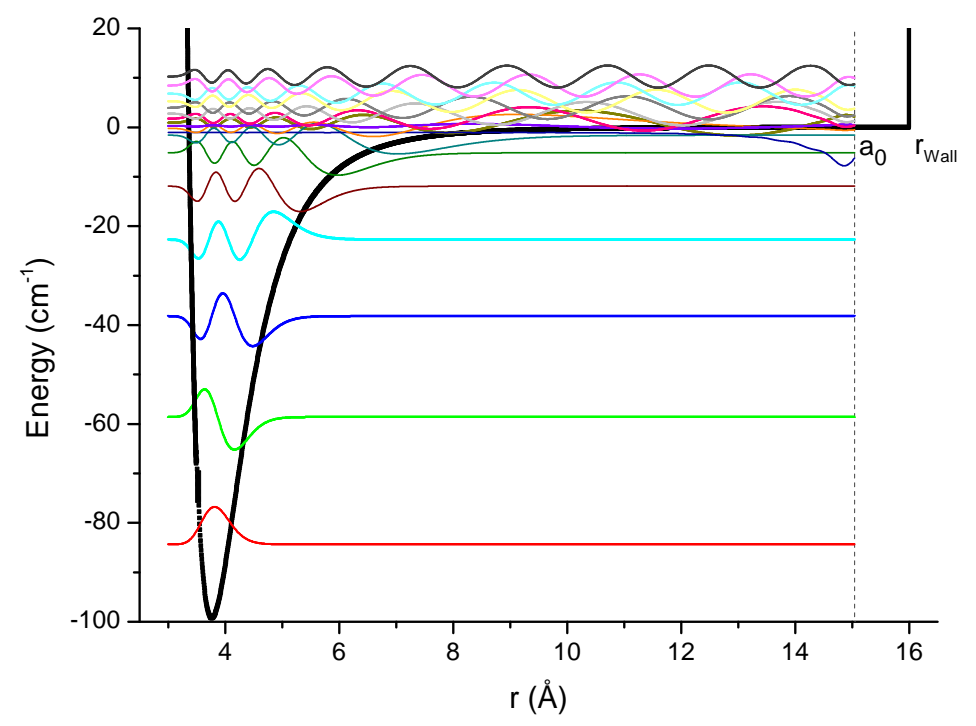

Fig. 2 Solutions of the inner region problem, $\chi_{n}^{0}$, for $\mathrm{Ar}_{2}$ showing both bound and continuum functions.

of the following Green's functions:

$$
\begin{array}{r}
\mathscr{G}_{i s}^{J}\left(r, r^{\prime}\right)=\frac{-1}{\lambda_{i s}^{J} \sin \left(\lambda_{i s}^{J} \delta a_{s}\right)} \\
\times\left\{\begin{array}{ll}
\cos \left(\lambda_{i s}^{J}\left(r^{\prime}-a_{s}\right)\right) \cos \left(\lambda_{i s}^{J}\left(r-a_{s-1}\right)\right) & a_{s-1} \leq r \leq r^{\prime} \\
\cos \left(\lambda_{i s}^{J}\left(r-a_{s}\right)\right) \cos \left(\lambda_{i s}^{J}\left(r^{\prime}-a_{s-1}\right)\right) & r^{\prime} \leq r \leq a_{s}
\end{array},\right.
\end{array}
$$

where $\delta a_{s}=a_{s}-a_{s-1}$. Then, defining $\mathbf{G}_{s}^{J}\left(r, r^{\prime}\right)$ as

$$
\mathbf{G}_{s}^{J}\left(r, r^{\prime}\right)=\mathbf{O}_{s}^{J} \mathscr{G}^{J}\left(\mathbf{O}_{s}^{J}\right)^{T}
$$

we can write down the expression for the propagation equation:

$$
\begin{array}{r}
a_{s} \mathbf{R}_{s}^{J}=\mathbf{G}_{s}^{J}\left(a_{s}, a_{s}\right)- \\
\mathbf{G}_{s}^{J}\left(a_{s}, a_{s-1}\right)\left(\mathbf{G}_{s}^{J}\left(a_{s-1}, a_{s-1}\right)+a_{s-1} \mathbf{R}_{s-1}^{J}\right)^{-1} \mathbf{G}_{s}^{J}\left(a_{s-1}, a_{s}\right),
\end{array}
$$

The size of each step in the iteration is variable, and dependent on the size of the last step. It obeys its own iteration equation, dependent on the derivative of the long-range potential used, in the following way ${ }^{85}$ :

$$
\delta a_{s+1}=\beta\left(\frac{1}{N} \sum_{i=1}^{N} \frac{\left(\lambda_{i, s}^{J}\right)^{2}-\left(\lambda_{i, s-1}^{J}\right)^{2}}{\delta a_{s}}\right)^{-1 / 3},
$$

where $i$ counts over the channels, $N$ is the number of channels and, $\beta$ is a control parameter which allows you to specify how many steps should be taken. $\beta$ is currently taken to be 0.1 . The variable step size ensures that for different potentials, the appropriate number of steps will be used to balance computation speed against accuracy. It also means that in the multi-channel case, channels which contribute different amounts can be treated differently. The initial step size is taken to be $0.1 \%$ of the distance from $a_{0}$ to $a_{p}$.

Next we introduce the Burke-Schey expansion, a specifc version of Eq. 11. In the Burke-Schey expansion, the matrices $\mathbf{s}^{J}$ and $\mathbf{c}^{J}$ have the following forms:

$$
s_{i i^{\prime}}^{J}=A_{i i^{\prime}}^{J} \cdot \sin \left(k_{i^{\prime}}^{J} r\right), \quad c_{i i^{\prime}}^{J}=B_{i i^{\prime}}^{J} \cdot \cos \left(k_{i^{\prime}}^{J} r\right),
$$

where

$$
A_{i i^{\prime}}^{J}=\sum_{p=0}^{p_{\max }} \alpha_{p i i^{\prime}}^{J} r^{-p}, \quad B_{i i^{\prime}}^{J}=\sum_{p=0}^{p_{\max }} \beta_{p i i^{\prime}}^{J} r^{-p},
$$

and the alpha and beta coefficients are derived from recurrence relations. For $\mathrm{Ar}_{2}$, the test system being studied, the long-range $\mathrm{Ar}_{2}$ potential is written as

$$
V_{i i^{\prime}}^{J}(r)=V_{i i^{\prime}}(r)+\frac{\hbar^{2} J(J+1)}{2 \mu r^{2}}=\sum_{\lambda=1}^{\lambda_{\max }} a_{\lambda i i^{\prime}} r^{-\lambda}+\frac{\hbar^{2} J(J+1)}{2 \mu r^{2}},
$$

and the diagonal coefficients $a_{\lambda i i}$ are obtained from Patkowski and Murdachaew 81 . Note in general $\lambda_{\max }$ can vary for different channels. The $\alpha_{p i i^{\prime}}^{J}$ and $\beta_{p i i^{\prime}}^{J}$ coefficients are then obtained from the following interdependent recurrence relations :

$$
\begin{array}{r}
\left(\left(k_{i}^{J}\right)^{2}-\left(k_{i^{\prime}}^{J}\right)^{2}\right) \alpha_{p i i^{\prime}}^{J}+((p-1)(p-2)-J(J+1)) \alpha_{p-2, i i^{\prime}}^{J} \\
+2 k_{i^{\prime}}^{J}(p-1) \beta_{p-1, i^{\prime}}^{J}=\sum_{i^{\prime \prime}=1}^{N} \sum_{\lambda=1}^{\lambda_{\max }} a_{i i^{\prime \prime}} \lambda \alpha_{p-\lambda-1, i^{\prime \prime} i^{\prime}}^{J},
\end{array}
$$

and

$$
\begin{array}{r}
\left(\left(k_{i}^{J}\right)^{2}-\left(k_{i^{\prime}}^{J}\right)^{2}\right) \beta_{p i i^{\prime}}^{J}+((p-1)(p-2)-J(J+1)) \beta_{p-2, i i^{\prime}}^{J} \\
-2 k_{i^{\prime}}^{J}(p-1) \alpha_{p-1, i i^{\prime}}^{J}=\sum_{i^{\prime \prime}=1}^{N} \sum_{\lambda=1}^{\lambda_{\max }} a_{i i^{\prime \prime} \lambda}^{J} \beta_{p-\lambda-1, i^{\prime \prime} i^{\prime}}^{J},
\end{array}
$$


where $N$ is the number of channels and $\lambda_{\max }$ is the largest value of $\lambda$ (with larger values increasing both accuracy and computation time). The derivatives of the $\mathbf{s}^{J}$ and $\mathbf{c}^{J}$ matrices also generate related recurrence relations, which can be derived by differentiating their power expansions.

Finally, the coefficients obtained from the recurrence relations are used to construct the asymptotic expansion. This expansion is combined with the R-matrix to form the K-matrix using eq. (12). From this K-matrix the eigenphases are then obtained, and from the eigenphases the cross sections are obtained.

\section{Results}

As an initial test of the inner region codes we intend to use as inputs to our new R-matrix code, we have looked for the so-called shape resonances trapped behind the centrifugal barrier in the rotationally excited $\mathrm{Ar}_{2}$ problem. Tests were performed for the rotational state $J=40$, which is sufficiently excited for the $\mathrm{Ar}_{2}$ system to not support any truly bound states. For this value of $J$, however, we would expect some quasibound states to exist behind a potential barrier, and for the Argon dimer potential of Patkowski and Murdachaew 81 , the peak of the centrifugal barrier is at $31.0389 \mathrm{~cm}^{-1}$. Calculations were performed by inputting this $\mathrm{Ar}_{2}$ potential on a grid both with Le Roy's diatomic code LEVEL 86 and with Duo.

The Duo results were obtained by computing bound and continuum energy levels both with and without a wall using a stabilisation procedure 87 . In the case of a wall energy levels were obtained in Duo up to $v=100$ at $J=40$ with the wall placed at various locations. A plot of various energy levels against wall location was then constructed, and the places on that plot where the energy levels appeared to lie on a horizontal line (i.e. where the energy did not vary with wall location) were used determine the energies of the shape resonances. This is because the continuum energies follow a particle-in-a-box type energy distribution, and so are dependent on the size of the 'box'. This is not the case for actual shape resonances.

A similar method was used to obtain the results without a wall, only instead of plotting energy against wall position, a plot of energy against the position of the end of the grid was used. Again, energy levels which did not vary with grid size were taken to be resonances, as opposed to a continuum state.

As Table 1 shows, all three methods give two resonance energy levels. Furthermore, all three methods agreed to an accuracy better than $0.0001 \mathrm{~cm}^{-1}$. The fact that in both Duo cases the horizontally aligned energies agreed with the LEVEL results was an encouraging indicator that resonance energies had indeed been found.

Table $1 \mathrm{Ar}_{2}$ shape resonance energies obtained with three different inner region solution methods at $J=40$. All energies are in units of $\mathrm{cm}^{-1}$.

\begin{tabular}{llll}
\hline & LEVEL & DUO & \\
\cline { 2 - 4 } $\mathrm{N}$ & & Wall & No wall \\
\hline 1 & 7.7126 & 7.7126 & 7.7126 \\
2 & 24.6178 & 24.6178 & 24.6178 \\
\hline
\end{tabular}

Figure 2 shows a further test of the inner region codes used as inputs for the R-matrix method, this time in the form of $\mathrm{Ar}_{2}$ inner region wavefunctions obtained for the inner region problem using the Patkowski and Murdachaew potential curve in Duo. The wall was placed at $16 \AA$ and the inner region boundary, $\mathrm{a}_{0}$ at $15.045 \AA$. We note that all bound state wavefunctions, except the highest one, are completely confined well inside our inner region boundary. The highest state's non-zero amplitude on the boundary is suspect and probably due to residual numerical issues. This means that the first 8 states make no contribution to the R-matrix on the boundary and can be dropped from consideration in the scattering region. For polyatomic systems, dropping the truly bound states from consideration should lead to substantial computational savings and could lead to the use of methods which do not compute wavefunctions for these states in the first place.

\section{Future Directions}

Our aim is to use the R-matrix formulation of scattering theory to study many-particle problems and, by extension, chemical reactions. As discussed above, the methodology should be particularly appropriate for ultra-low energy chemical reactions. Figure 3 illustrates how this should work for the prototypical reaction

$$
H_{2}+D^{+} \rightarrow H D+H^{+} \text {. }
$$

This exothermic reaction is likely to display significant resonance effects at very low energies. We note that Fig. 3 implies there is a change in coordinates, as the two asymptotes are most naturally represented in different sets of Jacobi coordinates. How precisely this is best achieved has to be determined, although one possibility would be to solve the inner region problem in one coordinate system, here the higher symmetry $\mathrm{H}_{2}-\mathrm{D}^{+}$Jacobi coordinates, for example, and then use a projection operator on the boundary to construct the R-matrix on the boundary for the other coordinates, in this case $\mathrm{HD}-\mathrm{H}^{+}$.

In practice, our proposed methodology is by no means limited to reactive scattering. Table 2 illustrates a number of the possibilities, again using the $\mathrm{H}_{2} \mathrm{D}^{+}$system as an example.

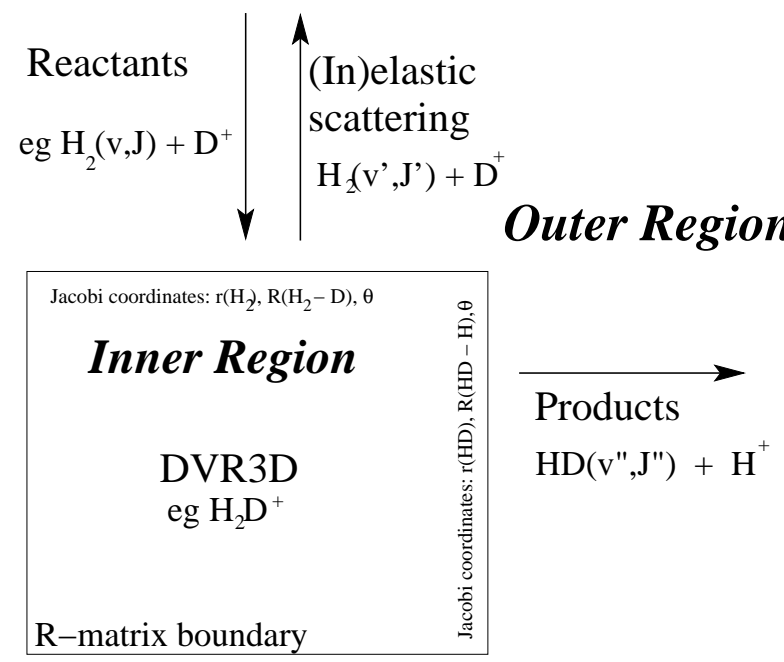

Fig. 3 Schematic illustrating of the use of the R-matrix method. 
Table 2 Processes that could be studied using a generalised R-matrix code: the $\mathrm{H}_{2} \mathrm{D}^{+}$system is simply used as an example, and not all possible processes or products are listed.

\begin{tabular}{ll}
\hline Process & Example \\
\hline Reactive scattering & $\mathrm{D}^{+}+\mathrm{H}_{2} \rightarrow \mathrm{HD}+\mathrm{H}^{+}$ \\
Photodissociation & $\mathrm{H}_{2} \mathrm{D}^{+}+h v \rightarrow \mathrm{HD}+\mathrm{H}^{+}$or $\mathrm{H}_{2}+\mathrm{D}^{+}$ \\
Photoassociation & $\mathrm{H}_{2}+\mathrm{D}^{+} \rightarrow \mathrm{H}_{2} \mathrm{D}^{+}+h v$ \\
Charge Exchange & $\mathrm{D}^{+}+\mathrm{H}_{2} \rightarrow \mathrm{D}+\mathrm{H}_{2}^{+}$ \\
Elastic collisions & $\mathrm{D}^{+}+\mathrm{H}_{2}(v, J) \rightarrow \mathrm{D}^{+}+\mathrm{H}_{2}(v, J)$ \\
Inelastic collisions & $\mathrm{D}^{+}+\mathrm{H}_{2}\left(v^{\prime \prime}, J^{\prime \prime}\right) \rightarrow \mathrm{D}^{+}+\mathrm{H}_{2}\left(v^{\prime}, J^{\prime}\right)$ \\
Predissociation & Not important for $\mathrm{H}_{2} \mathrm{D}^{+}$ \\
\hline
\end{tabular}

The division of space into two regions raises a number of interesting possibilities. So far our studies on Ar - Ar collisions have simply used the same potential energy curves in the inner and outer regions. However, the potential could be divided into two regions: an inner region potential which captures the full complexity of the reaction, a complex intermediate potential, and a long-range, outer region potential which can be represented using known multipolar forms for the dissociation fragment. Clearly, the two forms should match on the boundary. Standard quantum chemistry methodologies can be used to produce these potentials.

Another possibility is to include very weak effects only in the outer region. For example, first principles studies of molecular spectra routinely neglect hyperfine effects. However, these are important at ultra-low collision energies. In our proposed method one could re-couple hyperfine-free inner region wavefunctions on the boundary so that the outer region problem fully incorporates these effects. This approach has been successfully used to treat spin-orbit effects in electron - light atom collision problems for many years 88 . A similar approach could indeed be used to include spin-orbit effects, which are usually totally quenched in strongly bound closed-shell systems, but become important when dissociation occurs to open shell species, such as what happens in

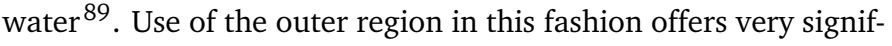
icant simplification compared to treating the full problem at all internuclear separations.

Experimental study of ultracold molecules is significantly enhanced through the use of electric and magnetic fields to tune resonances and thus increase production rates of the ultracold molecules $(90)$. Similarly, weak field effects could potentially be included in a similar fashion in the outer region. We note, however, that Zeeman effects have also recently been included in Duo. 91 .

\section{Conclusions}

The possibility of studying cold and ultracold collisions processes, and in particular chemical reactions, is one of the most interesting developments of this century. These experiments are stimulating whole new areas of scientific investigation, e.g. in quantum control, cold collisions, cold chemistry, accurate measurement, tests of fundamental physics, and more. Thus far most ultracold chemistry studies have been on alkali metal dimers. Looking to the future, the next major stride will involve reactions of chemically significant species and many atoms. Particularly important for novel aspects of ultracold physics will be the exploitation of res- onances: long-lived quasi-bound states of the compound system. The development of theoretical approaches, for example the one described in this paper, are essential for predicting, interpreting, and modelling this new physics. The R-matrix approach is designed to predict this interesting quantum behaviour and simulate and support experimental studies in a rigorous and flexible manner, both theoretically and computationally.

Our aim is to construct the harness code which links the inner and outer region segments. Initially this will be an atom-atom code used for testing numerical and algorithmic aspects of the procedure; some of these results are presented here. This work will be used to guide the developments for larger collision systems. The atom-atom code will also be used to study ultra-low energy collisions between systems being studied experimentally, starting with the $\mathrm{Ar}$ - Ar system mentioned above; this will allow us to explore the treatment of problems with coupled potentials and magnetic fields, and extend our work to other nuclear motion methods.

\section{Acknowledgments}

This project has received funding from the European Union's Horizon 2020 research and innovation programme under the Marie Sklodowska-Curie grant agreement No 701962 and from the EPSRC.

\section{References}

1 B. K. Stuhl, M. T. Hummon and J. Ye, Annu. Rev. Phys. Chem., 2014, 65, 501-518.

2 B. K. Stuhl, M. T. Hummon, M. Yeo, G. Quemener, J. L. Bohn and J. Ye, Nature, 2012, 492, 396-400.

3 E. S. Shuman, J. F. Barry and D. DeMille, Nature, 2010, 467, 820-823.

4 V. Zhelyazkova, A. Cournol, T. E. Wall, A. Matsushima, J. J. Hudson, E. A. Hinds, M. R. Tarbutt and B. E. Sauer, Phys. Rev. A, 2014, 89, 053416.

5 G. Quemener and P. S. Julienne, Chem. Rev., 2012, 112, 4949-5011.

6 J. D. Weinstein, R. deCarvalho, T. Guillet, B. Friedrich and J. M. Doyle, Nature, 1998, 395, 148-150.

7 V. Singh, K. S. Hardman, N. Tariq, M.-J. Lu, A. Ellis, M. J. Morrison and J. D. Weinstein, Phys. Rev. Lett., 2012, 108, 203201.

8 D. S. Jin and J. Ye, Chem. Rev., 2012, 112, 4801-4802.

9 C. Chin, R. Grimm, P. Julienne and E. Tiesinga, Rev. Mod. Phys., 2010, 82, 1225.

10 M. Mayle, G. Quemener, B. P. Ruzic and J. L. Bohn, Phys. Rev. A, 2013, 87, 012709.

11 S. Ospelkaus, K.-K. Ni, D. Wang, M. H. G. De Miranda, B. Neyenhuis, G. Quéméner, P. S. Julienne, J. L. Bohn, D. S. Jin and J. Ye, Science, 2010, 327, 853-857.

12 P. K. Molony, P. D. Gregory, Z. Ji, B. Lu, M. P. Köppinger, C. R. Le Sueur, C. L. Blackley, J. M. Hutson and S. L. Cornish, Phys. Rev. Lett., 2014, 113, 255301.

13 J. L. Bohn, Phys. Rev. A, 2001, 63, 052714.

14 J. M. Hutson, M. Beyene and M. L. González-Martínez, Phys. Rev. Lett., 2009, 103, 163201. 
15 T. Köhler, K. Góral and P. S. Julienne, Rev. Mod. Phys., 2006, 78, 1311.

16 T. Maier, I. Ferrier-Barbut, H. Kadau, M. Schmitt, M. Wenzel, C. Wink, T. Pfau, K. Jachymski and P. S. Julienne, Phys. Rev. A, 2015, 92, 060702(R).

17 P. S. Julienne, Nature, 2014, 507, 440-441.

18 J. P. D’Incao, H. Suno and B. D. Esry, Phys. Rev. Lett., 2004, 93, 123201.

19 T. Lompe, T. B. Ottenstein, F. Serwane, A. N. Wenz, G. Zürn and S. Jochim, Science, 2010, 330, 940-944.

20 F. Ferlaino, A. Zenesini, M. Berninger, B. Huang, H.-C. Nägerl and R. Grimm, Few-Body Systems, 2011, 51, 113-133.

21 Y. Wang and B. D. Esry, Phys. Rev. Lett., 2009, 102, 133201.

22 J. P. D’Incao and B. D. Esry, Phys. Rev. A, 2006, 73, 030703.

23 T. Kraemer, M. Mark, P. Waldburger, J. G. Danzl, C. Chin, B. Engeser, A. D. Lange, K. Pilch, A. Jaakkola, H.-C. Nägerl et al., Nature, 2006, 440, 315-318.

24 S. Knoop, F. Ferlaino, M. Mark, M. Berninger, H. Schöbel, H.C. Nägerl and R. Grimm, Nat. Phys., 2009, 5, 227-230.

25 J. von Stecher, J. P. D'Incao and C. H. Greene, Nat. Phys., 2009, 5, 417-421.

26 F. Ferlaino and R. Grimm, Physics, 2010, 3, 9.

27 M. Berninger, A. Zenesini, B. Huang, W. Harm, H.-C. Nägerl, F. Ferlaino, R. Grimm, P. S. Julienne and J. M. Hutson, Phys. Rev. Lett., 2011, 107, 120401.

28 I. Bloch, J. Dalibard and W. Zwerger, Rev. Mod. Phys., 2008, 80, 885.

29 M. Greiner, C. A. Regal and D. S. Jin, Nature, 2003, 426, 537540.

30 S. Jochim, M. Bartenstein, A. Altmeyer, G. Hendl, S. Riedl, C. Chin, J. Hecker Denschlag and R. Grimm, Science, 2003, 302, 2101-2103.

31 M. Bartenstein, A. Altmeyer, S. Riedl, S. Jochim, C. Chin, J. H. Denschlag and R. Grimm, Phys. Rev. Lett., 2004, 92, 120401.

32 T. Bourdel, L. Khaykovich, J. Cubizolles, J. Zhang, F. Chevy, M. Teichmann, L. Tarruell, S. Kokkelmans and C. Salomon, Phys. Rev. Lett., 2004, 93, 050401.

33 P. Pellegrini, M. Gacesa and R. Côté, Phys. Rev. Lett., 2008, 101, 053201.

34 S. C. Althorpe and D. C. Clary, Ann. Rev. Phys. Chem., 2003, 54, 493-529.

35 R. T. Pack and G. A. Parker, J. Chem. Phys., 1987, 87, 38883921.

36 J. M. Launay and M. Le Dourneuf, Chem. Phys. Lett., 1989, 163, 178-188.

37 P. Honvault, M. Jorfi, T. González-Lezana, A. Faure and L. Pagani, Phys. Rev. Lett., 2011, 107, 023201.

38 G. B. Pradhan, N. Balakrishnan and B. K. Kendrick, J. Phys. B: At. Mol. Opt. Phys., 2014, 47, 135202.

39 P. G. Burke, R-Matrix Theory of Atomic Collisions: Application to Atomic, Molecular and Optical Processes, Springer, 2011.

40 J. Tennyson, Phys. Rep., 2010, 491, 29-76.

41 P. Descouvemont and D. Baye, Rep. Prog. Phys., 2010, 73,
036301.

42 C. J. Bocchetta and J. Gerratt, J. Chem. Phys., 1985, 82, 13511362.

43 R. B. Walker and J. C. Light, Ann. Rev. Phys. Chem., 1980, 31, 401-433.

44 H. Y. Mussa and J. Tennyson, J. Chem. Phys., 1998, 109, 10885-10892.

45 G. H. Li and H. Guo, J. Mol. Struct. (Theochem), 2001, 210, 90-97.

46 A. G. Császár, E. Mátyus, L. Lodi, N. F. Zobov, S. V. Shirin, O. L. Polyansky and J. Tennyson, J. Quant. Spectrosc. Radiat. Transf., 2010, 111, 1043-1064.

47 N. F. Zobov, S. V. Shirin, L. Lodi, B. C. Silva, J. Tennyson, A. G. Császár and O. L. Polyansky, Chem. Phys. Lett., 2011, 507, 48-51.

48 J. R. Henderson and J. Tennyson, Chem. Phys. Lett., 1990, 173, 133-138.

49 J. R. Henderson, J. Tennyson and B. T. Sutcliffe, J. Chem. Phys., 1993, 98, 7191-7203.

50 M. J. Bramley, J. W. Tromp, T. Carrington and G. C. Corey, J. Chem. Phys., 1994, 100, 6175-6194.

51 J. J. Munro, J. Ramanlal and J. Tennyson, New J. Phys, 2005, 7, 196.

52 T. Szidarovszky, A. G. Csaszar and G. Czako, Phys. Chem. Chem. Phys., 2010, 12, 8373-8386.

53 S. Miller and J. Tennyson, Chem. Phys. Lett., 1988, 145, 117120.

54 R. Jaquet and T. Carrington, Jr., J. Phys. Chem. A, 2013, 117, 9493-9500.

55 A. A. Kyuberis, O. L. Polyansky, L. Lodi, J. Tennyson, R. I. Ovsyannikov and N. Zobov, Mon. Not. R. Astron. Soc., 2016.

56 T. Szidarovszky and A. G. Csaszar, Mol. Phys., 2013, 111, 2131-2146.

57 B. C. Silva, P. Barletta, J. J. Munro and J. Tennyson, J. Chem. Phys., 2008, 128, 244312.

58 S. N. Yurchenko, L. Lodi, J. Tennyson and A. V. Stolyarov, Comput. Phys. Commun., 2016, 202, 262-275.

59 J. Tennyson, M. A. Kostin, P. Barletta, G. J. Harris, O. L. Polyansky, J. Ramanlal and N. F. Zobov, Comput. Phys. Commun., 2004, 163, 85-116.

60 I. N. Kozin, M. M. Law, J. Tennyson and J. M. Hutson, Comput. Phys. Commun., 2004, 163, 117-131.

61 S. N. Yurchenko, W. Thiel and P. Jensen, J. Mol. Spectrosc., 2007, 245, 126-140.

62 A. Yachmenev and S. N. Yurchenko, J. Chem. Phys., 2015, 143, 014105.

63 O. L. Polyansky and J. Tennyson, J. Chem. Phys., 1999, 110, 5056-5064.

64 S. V. Shirin, O. L. Polyansky, N. F. Zobov, R. I. Ovsyannikov, A. G. Császár and J. Tennyson, J. Mol. Spectrosc., 2006, 236, 216-223.

65 O. L. Polyansky, N. F. Zobov, I. I. Mizus, L. Lodi, S. N. Yurchenko, J. Tennyson, A. G. Császár and O. V. Boyarkin, Phil. Trans. Royal Soc. London A, 2012, 370, 2728-2748. 
66 M. Pavanello, L. Adamowicz, A. Alijah, N. F. Zobov, I. I. Mizus, O. L. Polyansky, J. Tennyson, T. Szidarovszky and A. G. Császár, J. Chem. Phys., 2012, 136, 184303.

67 J. M. Hutson, New J. Phys, 2007, 9, 152.

68 P. Barletta, J. Tennyson and P. F. Barker, New J. Phys, 2009, 11, 055029.

69 P. D. Edmunds and P. F. Barker, Phys. Rev. Lett., 2014, 113, 183001.

70 J. Tennyson, L. Lodi, L. K. McKemmish and S. N. Yurchenko, J. Phys. B: At. Mol. Opt. Phys., 2016, 49, 102001.

71 D. A. Little and J. Tennyson, J. Phys. B: At. Mol. Opt. Phys., 2013, 46, 145102.

72 C. Bloch, Nucl. Phys., 1957, 4, 503.

73 J. Tennyson, J. Phys. B: At. Mol. Opt. Phys., 2004, 37, 10611071.

74 K. L. Baluja, P. G. Burke and L. A. Morgan, Computer Phys. Comm., 1982, 27, 299-307.

75 L. A. Morgan, Computer Phys. Comm., 1984, 31, 419-422.

76 J. C. Light and R. B. Walker, J. Chem. Phys., 1976, 65, 42724282.

77 V. M. Burke and C. J. Noble, Computer Phys. Comm., 1995, 85, 471-500.

78 P. G. Burke, C. J. Noble, A. G. Sunderland and V. M. Burke, Physica Scripta, 2002, T100, 55-63.

79 P. G. Burke and H. M. Schey, Phys. Rev., 1962, 126, 147.

80 M. Gailitis, J. Phys. B: At. Mol. Opt. Phys., 1976, 9, 843.

81 K. Patkowski, G. Murdachaew, C. M. Fou and K. Szalewicz, Mol. Phys., 2005, 103, 2031-2045.

82 A. T. Patrascu, C. Hill, J. Tennyson and S. N. Yurchenko, J. Chem. Phys., 2014, 141, 144312.

83 P.-O. Löwdin, J. Chem. Phys., 1950, 18, 365-375.

84 E. Anderson, Z. Bai, C. Bischof, S. Blackford, J. Demmel, J. Dongarra, J. Du Croz, A. Greenbaum, S. Hammarling, A. McKenney and D. Sorensen, LAPACK Users' Guide, Society for Industrial and Applied Mathematics, Philadelphia, PA, 3rd edn, 1999.

85 E. B. Stechel, R. B. Walker and J. C. Light, J. Chem. Phys., 1978, 69, 3518-3531.

86 R. J. Le Roy, LEVEL 8.0 A Computer Program for Solving the Radial Schrödinger Equation for Bound and Quasibound Levels, University of Waterloo Chemical Physics Research Report CP663, http://leroy.uwaterloo.ca/programs/, 2007.

87 H. S. Hazi, A. U.and Taylor, Phys. Rev. A, 1970, 1, 1109.

88 H. E. Saraph, Computer Phys. Comm., 1978, 15, 247-258.

89 O. V. Boyarkin, M. A. Koshelev, O. Aseev, P. Maksyutenko, T. R. Rizzo, N. F. Zobov, L. Lodi, J. Tennyson and O. L. Polyansky, Chem. Phys. Lett., 2013, 568-569, 14-20.

90 G. Quéméner and J. L. Bohn, Phys. Rev. A, 2013, 88, 012706.

91 M. Semenov, S. N. Yurchenko and J. Tennyson, J. Mol. Spectrosc., 2016. 